\title{
Environmental Temperature and
}

\section{Material Characterisation with Planar}

\section{Evanescent Microwave Sensors for}

\section{Environmental Analysis}

\section{Lavers, C}

http://hdl.handle.net/10026.1/16620

$10.1117 / 12.2585163$

All content in PEARL is protected by copyright law. Author manuscripts are made available in accordance with publisher policies. Please cite only the published version using the details provided on the item record or document. In the absence of an open licence (e.g. Creative Commons), permissions for further reuse of content should be sought from the publisher or author. 
Return to the Manage Active Submissions page at http://spie.org/submissions/tasks.aspx and approve or disapprove this submission. Your manuscript will not be published without this approval. Please contact authorhelp@spie.org with any questions or concerns.

\title{
Environmental Temperature and Material Characterisation with Planar Evanescent Microwave Sensors for Environmental Analysis
}

\author{
Christopher R. Lavers *a ${ }^{\mathrm{a}}$, Jonathan D. Fisk ${ }^{\mathrm{a}}$, Benjamin J.T. Lavers ${ }^{\mathrm{a}}$ \\ Dartmouth Centre for SeaPower and Strategy, Plymouth University at Britannia Royal Naval \\ College, College Way, Dartmouth, Devon, TQ6 0HJ;
}

\begin{abstract}
We discuss a microwave planar evanescent waveguide sensor, based on a patented technology demonstrator (2016), for a range of potential UAV platform skin-embedded waveguide sensing applications such as: refractive index sensing, temperature sensing, or surface corrosion. Evanescent microwave sensors can probe the near surface region from millimetre to metric scales, with minimal direct radiation into the external environment, ideal for evading detection, and able of detecting various dielectric or metal materials adjacent to the surface. This paper examines evanescent planar sensor use as an environmental evanescent sensor to both detect and quantify metal or dielectrics without direct contact, measuring material loss from chosen waveguide materials, and waveguide surface cladding materials. We also present microwave transmission for a range of temperature sensor configurations, with good sensitivity across a broad range of temperature-related applications, including temperature hysteresis. [1].
\end{abstract}

Keywords: evanescent, environmental temperature sensing, planar microwave sensor.

\section{INTRODUCTION}

We present experimental results here for fabrication of a range of microwave evanescent sensors based on planar optical waveguide principles, and the testing of several preliminary technology demonstrators, for scoping a wide range of potential sensing applications. Evanescent microwave and optical monitoring of the external surface skin of a UAV platform offers interesting design possibilities with low probability of intercept. Further possible microwave based evanescent sensors beyond the scope of this paper are discussed in our recent Plymouth University Research and Innovation Report [1]. Such evanescent microwave sensors are able to probe the near surface region to many centimetres, without any direct radiation of energy into the external environment, with potential for detection of various dielectric or metal materials of sensing interest. These devices follow similar optics planar sensor device designs already proven by the author [2]. This paper will look at the operation of an evanescent microwave sensor for refractive index sensing, an application of a device under patent [3], and presented in contemporary literature [4 - 5]. This paper will start by discussing the relevant theoretical background of integrated waveguide sensors before looking at the experimental methodology used. Results and a discussion of results are then presented, followed by some ideas for future work and the conclusions.

*christopher.lavers@plymouth.ac.uk ; phone +44 803 677218; fax +44 803677015 
Return to the Manage Active Submissions page at http://spie.org/submissions/tasks.aspx and approve or disapprove this submission. Your manuscript will not be published without this approval. Please contact authorhelp@spie.org with any questions or concerns.

\section{THEORETICAL BACKGROUND}

\section{1 Integrated Channel Microwave Wave Guide Sensor Devices}

Traditional microwave sensing methods mostly concern direct radiation of microwave energy emitted from a transmitter into the external environment, relying on either direct reflection of microwaves for detection or back-scattered from a point, surface, or volume source. Current radar sensing methods radiate microwave energy in a dispersive manner with cumulative additional losses from absorption and scattering, resulting in high attenuation and likely detection of transmitter action, which although not a problem for civilian activities could be disastrous for military operations. A possible innovative, and hitherto untried, microwave solution is provided through Attenuated Total Reflection (ATR) which has been used for several decades to couple optical radiation into thin film waveguide modes for evanescent sensing with prisms [6-8] and in more recent decades in planar waveguide geometries [9], with wave guide modes travelling within shallow waveguides, but sensing the near surface interface evanescently. Optical systems have a degree of limitation on the scale of environmental applications, as they only sense in close proximity to the surface region to a typical range of tens of wavelengths, ideal for bio-sensing applications, but not for large-scale phenomena. However, the flat geometry of planar or channel waveguides offers significant sensitivity for in-situ probing of the optical properties of attached surface layer properties and adjacent media because of the extended surface path length [9 - 10].

\subsection{Waveguide Advantages}

Practical microwave devices copy similar integrated optics planar sensor designs [2]. Attenuated Total Reflection is used widely to couple optical radiation into thin film waveguide modes [2] and in recent planar geometries [3-5]. Our microwave waveguides have a high index guide bounded by a lower aluminium metal plate with abrupt fixed field boundaries, and an upper surface to the air of lower permittivity which tapers the electric field into the superstrate medium (usually air or a cladding layer) on the order of wavelengths. Consider the transverse electric modes between the metal plates for TM (transverse magnetic with the electric field in the vertical y direction) with $\mathrm{Ex}=\mathrm{Ez}=0$ whilst Ey $\neq$ 0 shown in fig. 1.

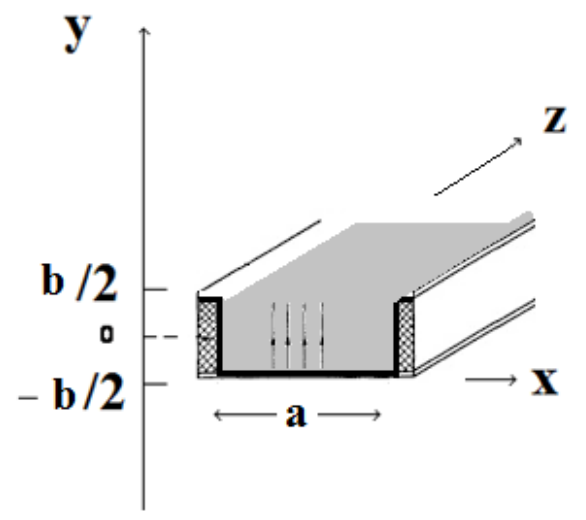

Fig. 1. Dielectric embedded waveguide inserted into metal waveguide cavity.

Modelling of the waveguide is determined accordingly with Maxwell's equations.

Since $\nabla \cdot E=0$ then $\mathrm{dEy} / \mathrm{dy}=0$ so clearly Ey is not a function of $\mathrm{y}$ and thus $\frac{d^{2} E_{y}}{d y^{2}}=0$

Deriving from Maxwell's equations: $\nabla^{2} E=-\varepsilon_{0} \mu_{0} \frac{d^{2} E}{d t^{2}}$ (1) which expanded is given by:

$$
\nabla^{2} E=\quad \frac{d^{2} E_{x}}{d x^{2}}+\frac{d^{2} E_{y}}{d y^{2}}+\frac{d^{2} E_{y}}{d z^{2}}=-\varepsilon_{0} \mu_{0} \frac{d^{2} E}{d t^{2}}
$$

This reduces to: $\frac{d^{2} E_{x}}{d x^{2}}+\frac{d^{2} E_{z}}{d z^{2}}=-\varepsilon_{0} \mu_{0} \frac{d^{2} E}{d t^{2}}$

With planar waveguides geometries offering high sensitivity for in-situ microwave probing near surface properties because of their path length. Microwave guiding is usually considered from a viewpoint they are a passive means of passing radiation along metal guides, not that guides may act as sensors in their own right. We select a waveguide 
Return to the Manage Active Submissions page at http://spie.org/submissions/tasks.aspx and approve or disapprove this submission. Your manuscript will not be published without this approval. Please contact authorhelp@spie.org with any questions or concerns.

surface region to access fields, increasing sensitivity to environment change. Robust dielectric embedded microwave planar waveguides can monitor changes in absorption of adjacent 'optically' thick surface layers, or changes in environmental microwave refractive index properties (due to the different metal / dielectric materials).

Fabrication was divided between planar waveguides some with embedded sensors, others a recess for refractive index measurement, or 1-D surface periodic gratings. Various guide materials were used; for demonstration purposes wax guides with Aluminium metal boundaries provided greatest design flexibility, regarded as 'leaky' Fabry Perot waveguides [4], loss increasing with length. Small air inclusions result in low/moderate scatter loss, but improved fabrication uses homogeneous materials i.e. (PTFE) [11] fig. 2.

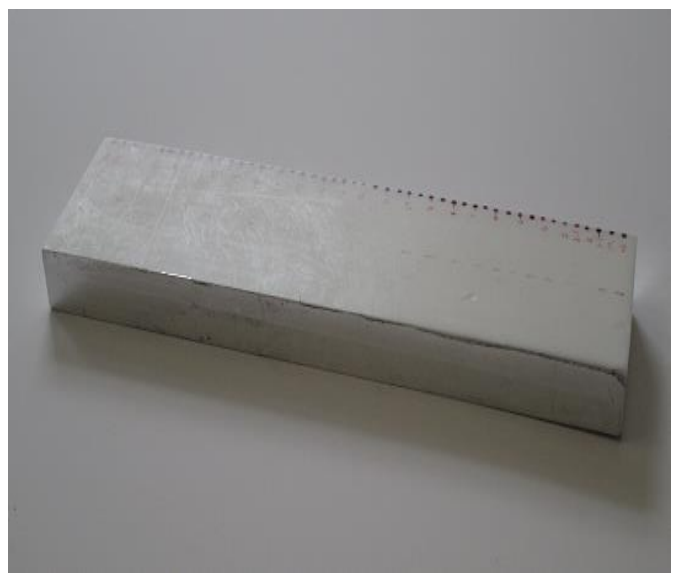

Fig. 2 Perspex waveguide.

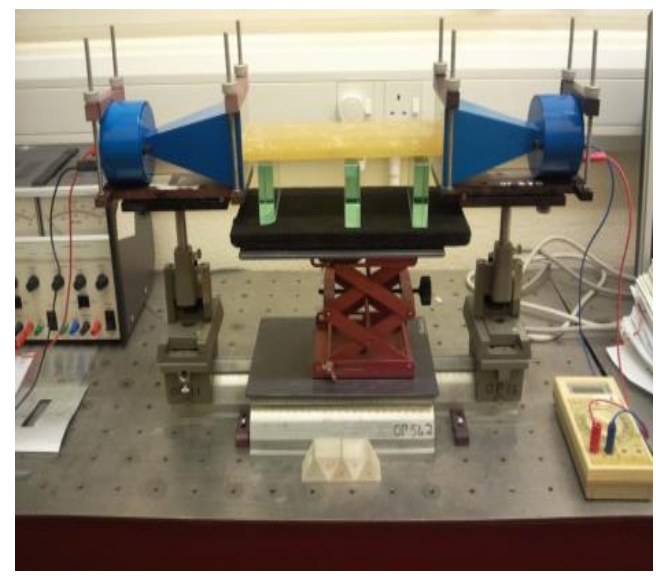

Fig. 3 Microwave input/output experimental coupling apparatus.

Wax was used in early designs, it is easy to pour into pre-fabricated moulds, sculpt into intricate designs, or add functionality. Guide dimensions are: $2 \mathrm{~cm}$ thick, $6.5 \mathrm{~mm}$ wide, $25.2 \mathrm{~cm}$ long for coupling to a microwave horn, fig. 3 ., In this figure a typical microwave cavity arrangement with transmitter to the left, receiver to the right (shown with waveguide installed) - TM mode. The horn source was powered by a Farnell LT30-2 dual power supply (output 10V nominally, checked with a Farnell DM141 multi-meter for stability monitoring. Receiver output voltage (mV) was monitored with digital multi-meters: a RS T100B and Fluke 89 IV data logger. Ambient temperature was monitored with a dual readout digital RS 427-461. A thermocouple unit (RS 610-067) measured - test samples, surfaces and waveguides. A Fluke 89 IV multimeter logged temperature readings during tests. A cavity shield $300 \times 150 \times 150$ mm was constructed, clad with aluminium plate with aluminium foil tape to cover the cavity minimising stray radiation leakage in the laboratory (fig. 4). 
Return to the Manage Active Submissions page at http://spie.org/submissions/tasks.aspx and approve or disapprove this submission. Your manuscript will not be published without this approval. Please contact authorhelp@spie.org with any questions or concerns.

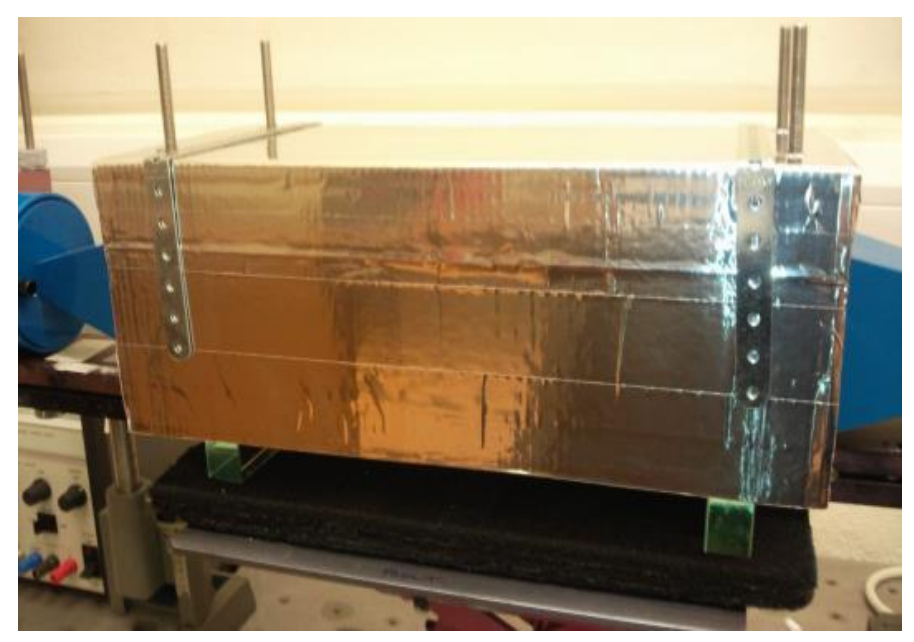

Fig. 4 Shielded waveguide cavity.

\section{RESULTS AND DISCUSSION}

Waveguide Materials Used:

Six different prospective waveguide materials were investigated, with maximum output recorded in fig. $\mathbf{5}$, for a practical device length, typically about $25 \mathrm{~cm}$. yielding maximum guide output per centimetre length. Fig. 5 of output waveguide transmission clearly indicates that both PTFE and Perspex ${ }^{\mathrm{TM}}$ waveguides provide the best choices in terms of reduced waveguide loss, however, wax was found to be the easiest material to work with and shape for overall initial designs, and was generally used in most moulded waveguide work [9].

\section{Waveguide Cladding Materials:}

Different materials were examined placing $6.5 \mathrm{~cm}$ square targets on a PTFE guide surface with the near edge placed along the guide's centreline. These materials are then plotted in terms of overall increasing relative microwave transmission compared with air superstrate with the exception of a common aviation alloy (Aluminium 2024-T3) response before and after immersion in a standard Vernier saline solution. Results for different waveguide cladding materials are shown in fig. 6.

\section{Different waveguides best microwave transmission $\mathrm{mV}$ per centimetre 1. Resin 2. Wax with recessed well 3. Polypropylene 4. Wax 5. PTFE 6. Perspex}

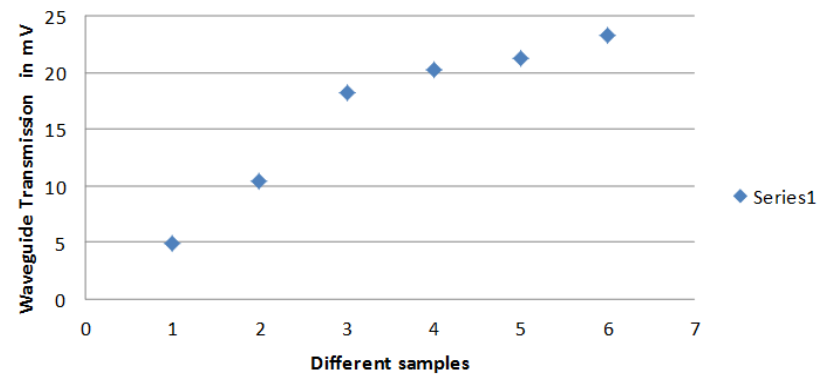

Fig. 5 Different Waveguide materials. 
Return to the Manage Active Submissions page at http://spie.org/submissions/tasks.aspx and approve or disapprove this submission. Your manuscript will not be published without this approval. Please contact authorhelp@spie.org with any questions or concerns.

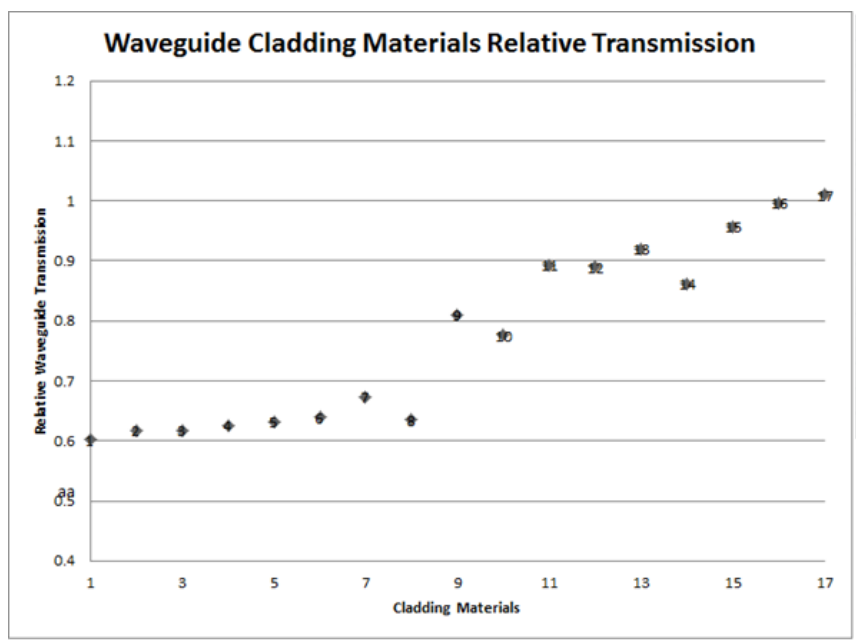

1 Al foil (bacofoil)
2 Palladium leaf
3 Carbon Fibre
4 Silver leaf
5 Gold leaf
6 Copper leaf
7 Al foil
8 Aviation Alloy $20243-T 3$
9 Aviation Alloy 20243-T3 1 week in standard saline solution
10 Brass YO 446
11 ROWI Glass slide
12 Copper (thick foil)
13 PTFE
14 Extra Mature British Cheddar
15 Lasage (dry)
16 Chocolate
17 Dry Cracker (Jacob)

Fig 6. Relative waveguide transmission for a range of different cladding materials.

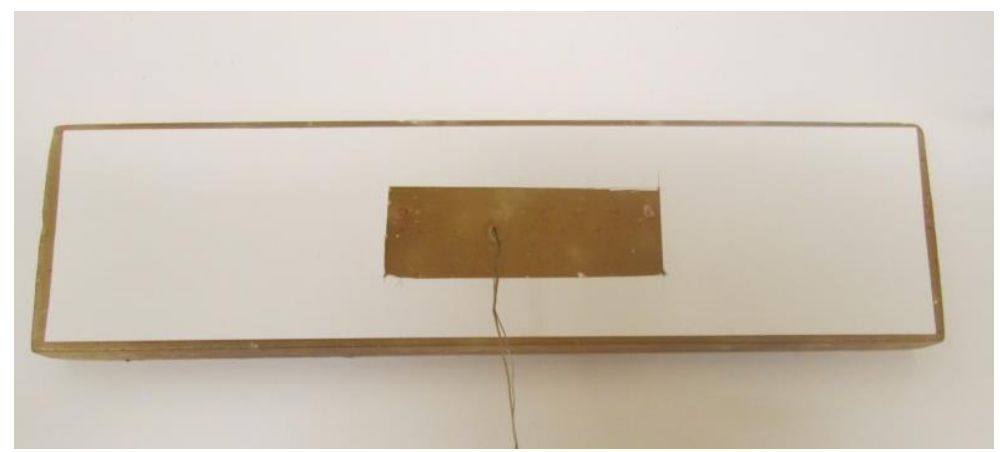

Fig. 7 Embedded thermocouple sensor.

Thermal Measurements: waveguide output changes with forced heating. A fabricated wax guide with a small exposed area whilst the rest is covered with a reflective non-metal 'mask' as metal will make a 'leaky waveguide' into a highly guiding one. A thermocouple was embedded in the waveguide (fig. 7). A heat source was placed approximately $20 \mathrm{~cm}$ above the waveguide mask for 2100 seconds, whilst monitoring TM radiation waveguide output. Ambient temperature rose $1.6^{\circ} \mathrm{C}$ and the waveguide temperature $+5.4^{\circ} \mathrm{C}$ (fig.8a) plotted over time. The source was then switched off, cooling over 5250s with the waveguide temperature falling to ambient. Hysteresis is seen in the waveguide output response fig.8b. 
Return to the Manage Active Submissions page at http://spie.org/submissions/tasks.aspx and approve or disapprove this submission. Your manuscript will not be published without this approval. Please contact authorhelp@spie.org with any questions or concerns.

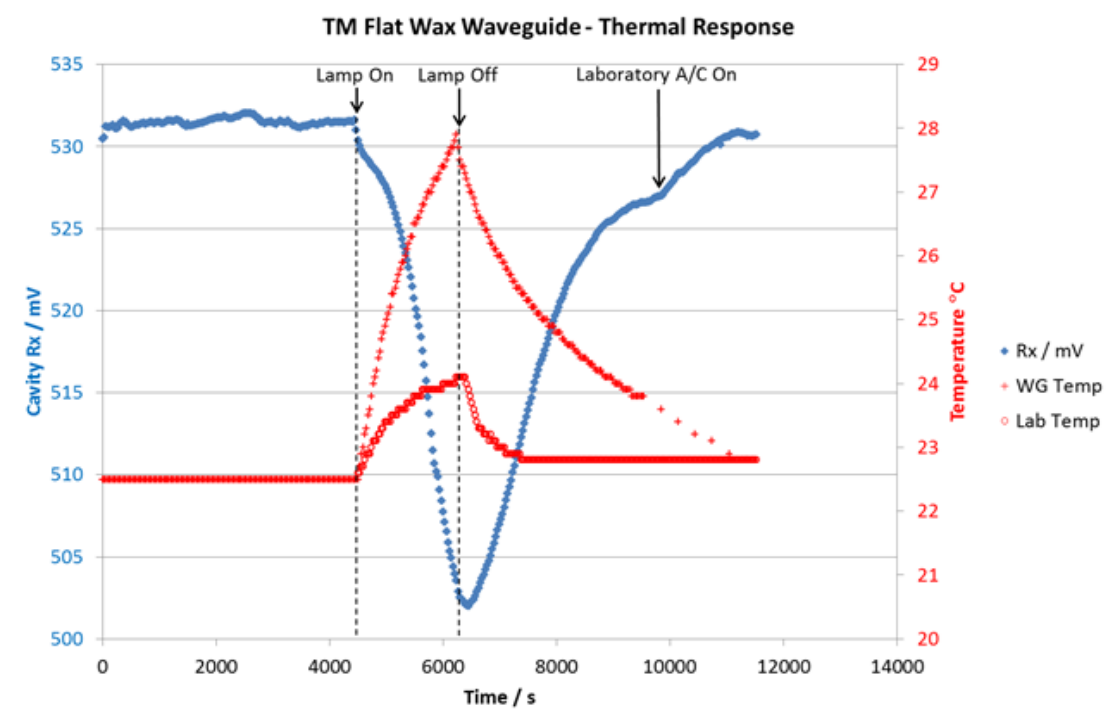

Fig. 8a: TM Wax guide Heating/Cooling Cycle.

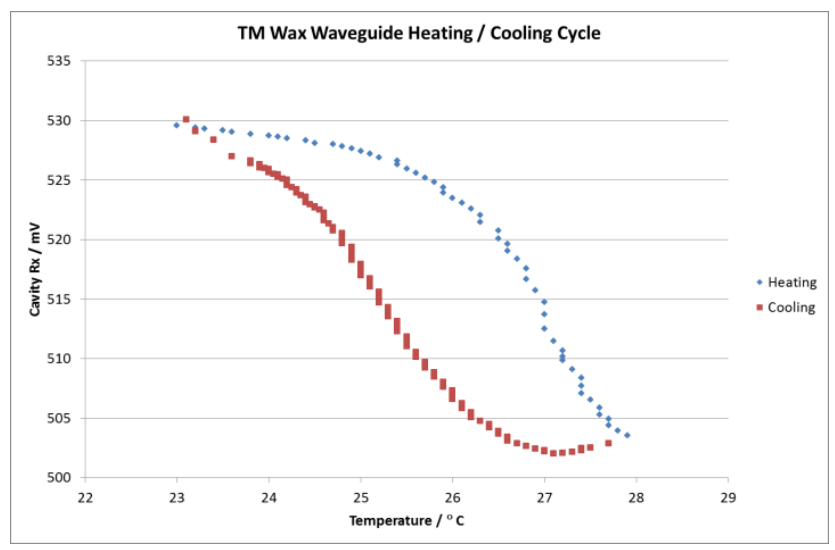

Fig.8b: Observed Waveguide Temperature Hysteresis of Voltage vs Temperature.

The waveguide has a repeatable and predictable behaviour response to direct heating which may be modelled. The test described here can be repeated and run for longer timescales so a constant elevated temperature could be achieved once equilibrium between the waveguide and the ambient air is reached for a given heating load. Extension of such testing might include construction of an enclosure with heating and cooling systems allowing full control of the waveguide environment temperature. This could be used to yield a relationship between the cavity output signal and waveguide temperature at a given ambient temperature. For the result presented here, hysteresis in the heating and cooling cycle was identified showing the effect from the ambient environment on heat transfer rates to and from the waveguide. This section demonstrates a temperature responsive material can provide a suitable temperature dependent waveguide sensor with a variety of surface relief masks and surface embedded sensors, some of which are readily available for purchase [12].

Ice Melting: Studies were also conducted on phase change: as solid to liquid phase changes are present during icing, and is highly relevant to manned and unmanned aerial vehicle (UAV) platform surfaces. Small glass pots were filled with $4 \mathrm{ml}$ deionised water, a thermocouple immersed, and then frozen. Pots were then placed on a waveguide, and either Transvers Magnetic TM or Transverse Electric (TE) mode outputs recorded whilst the ice warmed / melted. 


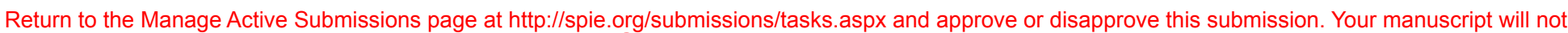
be published without this approval. Please contact authorhelp@spie.org with any questions or concerns.

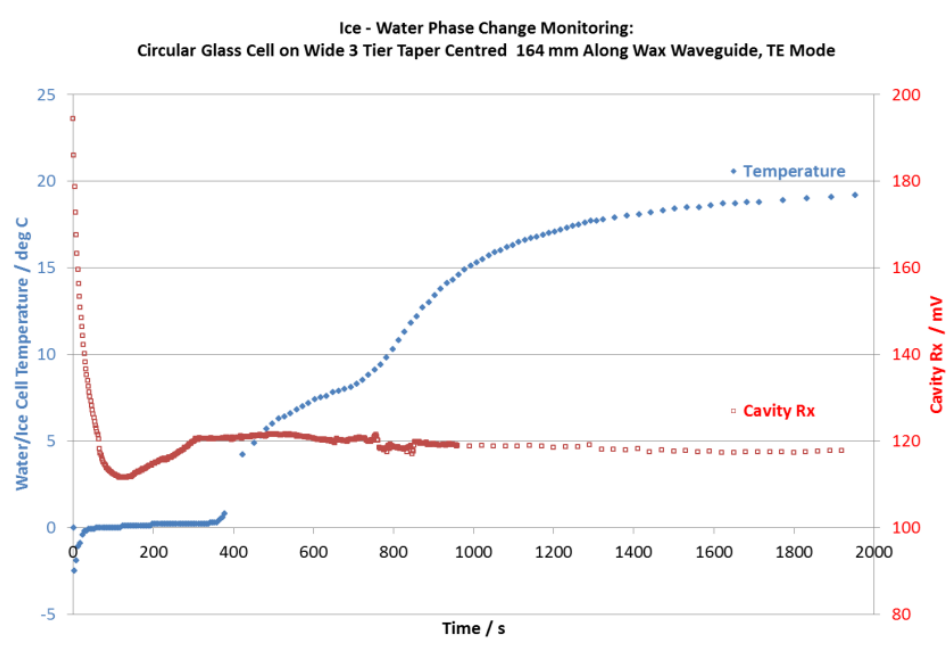

Fig. 9: Ice - Water phase change monitoring.

Temperature starts at $-2.5^{\circ} \mathrm{C}$ after guide installation. As the test progresses phase transition: ice to water occurs, holding about $0^{\circ} \mathrm{C}$ for c. $280 \mathrm{~s}$. Subsequently the pot warms to ambient $22.5^{\circ} \mathrm{C}$. Cavity output rapidly falls, reaching minimum at phase change, followed by a rise to a higher steady temperature (fig. 9).

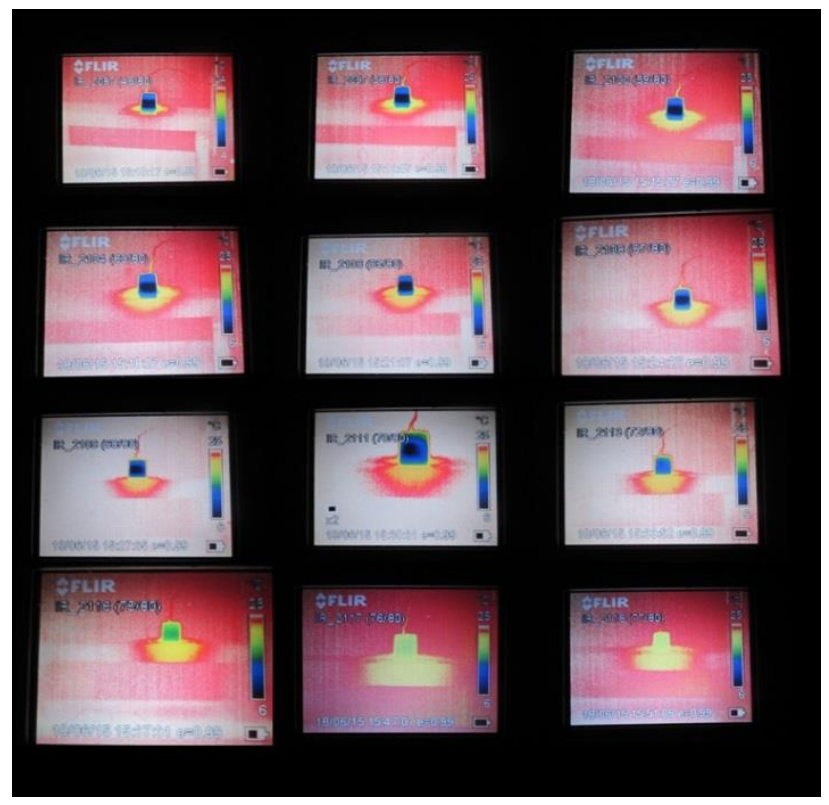

Fig. 10 FLIR images of frozen water pot left to right then down at regular time intervals showing diffusion related cooling of the frozen pot on the wax guide, and the subsequent warming and melting of the water.

The signal 'dip' is likely a result of a complex situation where a cavity experiences a combination of chilling when a cold pot cools a guide, and changing dielectric behaviour as ice melts to water, altering absorbed evanescent microwave energy. Pure water dielectric loss at $10 \mathrm{GHz}$ (near our frequency) and close to common navigation related-radar frequencies is maximum close to $0^{\circ} \mathrm{C}$. A Forward Looking Infra Red (FLIR) E320 radiometrically calibrated thermal camera sequence (fig. 10) shows heating / melting of a frozen pot, (top row left to right then down at 3 minute intervals). Initially ice appears cold 'black', whilst the guide cools over time with "cold diffusion" transferred from the pot into the waveguide. The large difference in the real part of the dielectric constant for radiation around $10 \mathrm{GHz}$ at the melting point of ice is presented here in fig. 11 [13]. 
Return to the Manage Active Submissions page at http://spie.org/submissions/tasks.aspx and approve or disapprove this submission. Your manuscript will not be published without this approval. Please contact authorhelp@spie.org with any questions or concerns.

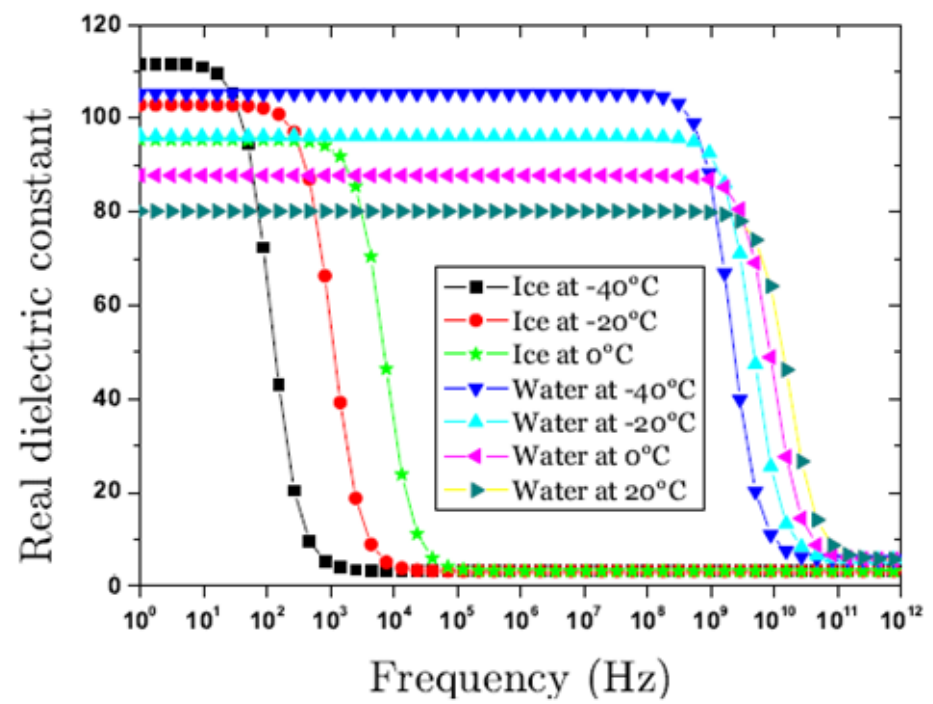

Figure 11: Complex behaviour of the real part of dielectric constant of water and ice at $10 \mathrm{GHz}$ and other frequencies as a function of temperature [13].

The dielectric loss of pure water at around $10 \mathrm{GHz}$ (very close to the frequency of the microwave source used here) shows a maximum very close to $0^{\circ} \mathrm{C}$ and consequently an anticipated rapid fall and then recovery in output transmission [14]. Therefore, during the phase transition when melt water forms and can gather as a film beneath any floating ice in the test cell or on the waveguide surface there is likely to be a minimum in the signal response whilst the dielectric loss is at a maximum. The prominence of the trough is somewhat less with the 'drop' tests compared with a frozen cell owing to the much smaller volume of ice/water absorbing the microwave radiation.

\section{CONCLUSIONS}

Planar waveguides allows microwave output temperature response to be examined evanescently. The waveguide temperature response demonstrated clear hysteresis behaviour. Microwave output transmission also shows loss as a function of target material, clearly showing that PTFE or Perspex provide the lowest loss per centimetre, and exhibiting cladding material selectivity. In other work metal targets have also been detected through layers of sand as a function of depth [5]. Sensitivity over time for small water volume provides good output correlation with temperature. Thermal imagery of a combined pot/guide shows guide cooling accompanies cell warming. However, separating the contributions of both cooling / heating is difficult.

\section{Acknowledgements}

Dr Paul Tiltman of the Research and Innovations Team, Mr Benjamin Lavers for several different waveguide fabrications, and Mr Mike Sloman for PTFE / Perspex fabrication. 
Return to the Manage Active Submissions page at http://spie.org/submissions/tasks.aspx and approve or disapprove this submission. Your manuscript will not be published without this approval. Please contact authorhelp@spie.org with any questions or concerns.

\section{REFERENCES}

[1] Lavers, C.R. and Fisk, J.D., 'A new class of evanescent micro wave sensor for various applications, 'Innovation Report”, December 2015.

[2] Lavers, C.R., 'Planar Optical Waveguides for Civil and Military Applications', Sensors and Their Applications XV, Journal of Physics: Conference Series 178, Proceedings doi: 1088/1742-6596/178/1/012010 ISSN: 1742-6588, (2009).

[3] Lavers, C.R. et. al., Microwave Sensor for Various Applications, Plymouth University (Patent Office Number: GB1612057.8 09/Jan 2017).

[4] Lavers, C.R. and Fisk, J.D., 'A New Planar Evanescent Microwave Sensor for Refractive Index Measurement', Proceedings of Sensors and Their Applications XVIII, QMUL London, (2016).

[5] Lavers, C.R. and Fisk, J.D., 'An Evanescent Channel Microwave Waveguide Sensor for Metal Detection through Variable Sand Depth' International Journal of Recent Trends in Electrical \& Electronics Engg., Dec., 2017. ISSN: 22316612, (2017).

[6] Otto, A., 'Excitation of non-radiative surface plasma waves in silver by the method of frustrated total reflection', Zeitschrift fur Physik, 216(4):398-410, (1968).

[7] Kretschmann, E., and Raether, H., 'Radiative Decay of Non Radiative Surface Plasmons Excited by Light', Verlag der Zeitschrift fur Naturforschung (a) 23:2135-2136, (1968).

[8] Lavers, C.R., and Sambles, J.R., 'Use of mode mixing to determine the optic tensor configuration of a thin ferroelectric liquid crystal layer', Liquid Crystals, 8, 577-585, (1990).

[9] Lavers, C.R., Piraud, C., Wilkinson, J.S., Brust, M., O Dwyer, K., and Schiffrin, D.J., 'Electrochemically controlled optical waveguide sensors', Proceedings of Optical Fiber Sensors 9 in Florence, May 4 - 6th, (1993).

[10] Lavers, C.R., Harris, R.D., Hao, S., Wilkinson, J.S., O Dwyer, K., Brust, M., and Schiffrin, D.J., "Electrochemically-controlled waveguide-coupled surface plasmon sensing", Journal of Electroanalytical Chemistry, Vol.387, No.1, pp.2-11, (1995).

[11] Lavers, C., Fisk, J., "An Evanescent Channel Microwave Waveguide Sensor for Metal Detection through Variable Sand Depth", International Journal of Recent Trends in Electrical \& Electronics Engg., Dec., 2017. ISSN: 22316612 ,Volume 6, Issue 1: 08-16, (2017)..

[12] Thermo Electric ${ }^{\mathrm{TM}}$ http://www.te-direct.com/products/semiconductor/

[13] Dielectric capacity, liquid water content, and pore structure of thawing-freezing materials; Antonin Fabbri, Teddy Fen-Chong*, Olivier Coussy; Institut Navier, LMSGC 2 all'ee Kepler - 77420 Champs-surMarne, France]. http://sarah.antonin.free.fr/cold.pdf]

[14] http://www1.lsbu.ac.uk/water/microwave_water.html Dielectric loss graph Page Author: Martin Chaplin. Equations plotted out originally from: T. Meissner and F. J. Wentz, The complex dielectric constant of pure and sea water from microwave satellite observations, IEEE Trans. Geosci. Remote Sensing 42 1836-1849, (2004). 